\title{
Atomic data for astrophysics: Ni XII ${ }^{\star}$
}

\author{
G. Del Zanna ${ }^{1}$ and N. R. Badnell ${ }^{2}$ \\ 1 DAMTP, Centre for Mathematical Sciences, University of Cambridge, Wilberforce Road, Cambridge, CB3 0WA, UK \\ e-mail: g.del-zanna@damtp.cam.ac.uk \\ 2 Department of Physics, University of Strathclyde, Glasgow, G4 0NG, UK
}

Received 16 September 2015 / Accepted 5 November 2015

\section{ABSTRACT}

\begin{abstract}
We present new large-scale R-matrix (up to $n=4$ ) scattering calculations for the electron collisional excitation of Cl-like Ni XII. We used the intermediate-coupling frame transformation method. We compare predicted and observed line intensities using laboratory and solar spectra, finding good agreement for all the main soft X-ray lines. With the exception of the three strongest transitions, large discrepancies with previous estimates are found, especially for the decays from the lowest $3 \mathrm{~s}^{2} 3 \mathrm{p}^{4} 3 \mathrm{~d}$ levels. This includes the forbidden UV lines. The atomic data for the $n=4$ levels are the first to be calculated. We revise previous experimental energies, and suggest several new identifications. We point out the uncertainty in the wavelength of the $3 \mathrm{~s}^{2} 3 \mathrm{p}^{5}{ }^{2} \mathrm{P}_{1 / 2}-3 \mathrm{~s}^{2} 3 \mathrm{p}^{4} 3 \mathrm{~d}^{2} \mathrm{D}_{3 / 2}$ transition, which is important for density diagnostics.
\end{abstract}

Key words. atomic data - techniques: spectroscopic - Sun: corona - line: identification

\section{Introduction}

Lines from Cl-like Ni XII are prominent in the soft X-rays and EUV (Gabriel et al. 1965). Several forbidden lines are also observed in the visible and UV part of the solar spectrum (Edlen $\&$ Smitt 1978). Nickel lines are also prominent in fusion plasma (see, e.g. Mattioli et al. 2004). Accurate atomic data are needed for this ion.

The most recent calculations for excitation by electron impact were carried out by Matthews et al. (1998). These authors included only the lowest 14 LS target states (31 fine-structure levels) of the $3 s^{2} 3 p^{5}, 3 s 3 p^{6}$, and $3 s^{2} 3 p^{4} 3 d$ configurations in their close-coupling (CC) expansion. The CIV3 program was used to calculate the wave functions in LS coupling, and the R-matrix method (Berrington et al. 1995) was used to calculate the collision stengths in LS coupling. The $j$-resolved collision stengths were obtained using the JAJOM program (Saraph 1978).

The excitation rates calculated by Matthews et al. (1998) were supplemented with A-values and included in the CHIANTI database v. 2 (Landi et al. 1999). They are still in the current v. 8 of CHIANTI (Del Zanna et al. 2015). We note, however, that the excitation rates included in CHIANTI originated from a database that was held at Queen's University of Belfast, and that some differences between the values in the database and those tabulated in Matthews et al. (1998) are present (E. Landi, priv. comm.).

Our recent large-scale atomic calculations for Cl-like Fe X (Del Zanna et al. 2012) have shown a significant improvement, compared to the previous smaller calculations (Del Zanna et al. 2004), in particular for the forbidden lines (see Del Zanna et al. 2014) and the decays from the $3 s^{2} 3 p^{4} 3 d{ }^{4} D_{7 / 2,5 / 2}^{e}$, with typical changes larger than $50 \%$. Del Zanna et al. (2012) in-

* The full dataset is available at our APAP website (www . apapnetwork.org) and at the CDS via anonymous ftp to cdsarc.u-strasbg.fr (130.79.128.5) or via http://cdsarc.u-strasbg.fr/viz-bin/qcat?J/A+A/585/A118 cluded the full set of 32 configurations up to $n=4$ in the configuration-interaction (CI) expansion and $218 \mathrm{LS}$ terms in the CC expansion, i.e. much larger than the Matthews et al. (1998) calculation.

The aim of this paper is to present a large-scale calculation for Ni XII along the lines of the Fe X one, assess differences with the previous calculation, and benchmark the data against observations.

\section{Atomic structure}

The atomic structure calculations were carried out using the AUTOSTRUCTURE program (Badnell 2011) which constructs target wavefunctions using radial wavefunctions calculated in a scaled Thomas-Fermi-Dirac statistical model potential with a set of scaling parameters.

For the CI expansion we adopted the same set of 32 configurations up to $n=4$ used for Fe X (Del Zanna et al. 2012), namely $3 \mathrm{~s}^{2} 3 \mathrm{p}^{5}, 3 \mathrm{~s} 3 \mathrm{p}^{6}, 3 \mathrm{~s}^{2} 3 \mathrm{p}^{4} 3 \mathrm{~d}, 3 \mathrm{~s}^{2} 3 \mathrm{p}^{3} 3 \mathrm{~d}^{2}, 3 \mathrm{~s} 3 \mathrm{p}^{5} 3 \mathrm{~d}, 3 \mathrm{p}^{6} 3 \mathrm{~d}, 3 \mathrm{p}^{5}$ $3 \mathrm{~d}^{2} 3 \mathrm{~s}^{2} 3 \mathrm{p}^{4} 4 \mathrm{l}(\mathrm{l}=\mathrm{s}, \mathrm{p}, \mathrm{d}, \mathrm{f}), 3 \mathrm{~s} 3 \mathrm{p}^{5} 4 \mathrm{l}(\mathrm{l}=\mathrm{s}, \mathrm{p}, \mathrm{d}, \mathrm{f}), 3 \mathrm{~s}^{2} 3 \mathrm{p}^{3} 3 \mathrm{~d} 4 \mathrm{l}$ $(1=s, p, d, f), 3 p^{6} 41,3 p^{5} 3 d 4 l(l=s, p, d, f)$. The scaling parameters $\lambda_{\mathrm{nl}}$ for the potentials in which the orbital functions are calculated are 1s: $1.40650,2 \mathrm{~s}: 1.12427,2 \mathrm{p}: 1.06865,3 \mathrm{~s}: 1.17240,3 \mathrm{p}$ : 1.14400, 3d: 1.17063, 4s: 1.17191, 4p: 1.14250, 4d: 1.15475, 4f: 1.28158 .

Ions such as Ni XII are particularly complex because of the strong mixing between levels having the same $J$-value and parity. The mixing between fine-structure levels substantially changes depending on the configurations included in the target representation, and, more importantly, on the relative position of the levels, which we improved with the term energy correction (TEC) method, introduced by Zeippen et al. (1977) and Nussbaumer \& Storey (1978).

Unfortunately, only very few level energies are well known. We list the few experimental level energies $E_{\text {exp }}$ in Table 1. From these, we obtained a set of "best guess" energies $E_{\text {best }}$ by linear interpolation. We then used the $E_{\text {best }}$ values to obtain the TEC 
Table 1. Target level energies.

\begin{tabular}{|c|c|c|c|c|c|c|}
\hline$i$ & Conf. & Mixing & LSJ & $E_{\text {exp }}$ & $E_{\mathrm{TEC}}$ & \\
\hline 1 & $3 s^{2} 3 p^{5}$ & $(96 \%)$ & ${ }^{2} \mathrm{P}_{3 / 2}$ & 0.0 & 0.0 & \\
\hline 2 & $3 s^{2} 3 p^{5}$ & $(96 \%)$ & ${ }^{2} \mathrm{P}_{1 / 2}$ & 23629.0 & $24444.0(-815)$ & \\
\hline 3 & $3 s 3 p^{6}$ & $(75 \%)+27(\mathrm{c} 322 \%)$ & ${ }^{2} \mathrm{~S}_{1 / 2}$ & 338615.0 & $338871.0(-256)$ & \\
\hline 4 & $3 s^{2} 3 p^{4} 3 d$ & $(90 \%)$ & ${ }^{4} \mathrm{D}_{5 / 2}$ & 452755.0 & $449645.0(3110)$ & $\mathrm{T}$ \\
\hline 5 & $3 s^{2} 3 p^{4} 3 d$ & $(93 \%)$ & ${ }^{4} \mathrm{D}_{7 / 2}$ & 454036.0 & $450723.0(3313)$ & $\mathrm{T}$ \\
\hline 6 & $3 s^{2} 3 p^{4} 3 d$ & $(88 \%)$ & ${ }^{4} \mathrm{D}_{3 / 2}$ & - & 451114.0 & \\
\hline 7 & $3 s^{2} 3 p^{4} 3 d$ & $(88 \%)$ & ${ }^{4} \mathrm{D}_{1 / 2}$ & - & 453828.0 & \\
\hline 8 & $3 s^{2} 3 p^{4} 3 d$ & $(46 \%)+30(40 \%)$ & ${ }^{2} \mathrm{P}_{1 / 2}$ & - & 482248.0 & \\
\hline 9 & $3 s^{2} 3 p^{4} 3 d$ & $(91 \%)$ & ${ }^{4} \mathrm{~F}_{9 / 2}$ & 485570.0 & $483901.0(1669)$ & $\mathrm{T}$ \\
\hline 10 & $3 s^{2} 3 p^{4} 3 d$ & $(78 \%)+18(10 \%)$ & ${ }^{4} \mathrm{~F}_{7 / 2}$ & 492790.0 & $490839.0(1951)$ & $\mathrm{T}$ \\
\hline 11 & $3 s^{2} 3 p^{4} 3 d$ & $(39 \%)+16(22 \%)+28(29 \%)$ & ${ }^{2} \mathrm{P}_{3 / 2}$ & - & 493404.0 & \\
\hline 12 & $3 s^{2} 3 p^{4} 3 d$ & $(93 \%)$ & ${ }^{4} \mathrm{P}_{1 / 2}$ & - & 496677.0 & \\
\hline 13 & $3 s^{2} 3 p^{4} 3 d$ & $(30 \%)+15(29 \%)+31(19 \%)+25(12 \%)$ & ${ }^{2} \mathrm{D}_{3 / 2}$ & - & 496904.0 & \\
\hline 14 & $3 s^{2} 3 p^{4} 3 d$ & $(94 \%)$ & ${ }^{4} \mathrm{~F}_{5 / 2}$ & 495000.0 & $497668.0(-2668)$ & $\mathrm{T}$ \\
\hline 15 & $3 s^{2} 3 p^{4} 3 d$ & $(62 \%)+13(12 \%)+16(13 \%)$ & ${ }^{4} \mathrm{~F}_{3 / 2}$ & - & 501278.0 & \\
\hline 16 & $3 s^{2} 3 p^{4} 3 d$ & $(54 \%)+11(12 \%)+28(11 \%)$ & ${ }^{4} \mathrm{P}_{3 / 2}$ & 504300.0 & $506890.0(-2590)$ & $\mathrm{T}$ \\
\hline 17 & $3 s^{2} 3 p^{4} 3 d$ & $(77 \%)$ & ${ }^{4} \mathrm{P}_{5 / 2}$ & - & 508755.0 & \\
\hline 18 & $3 s^{2} 3 p^{4} 3 d$ & $(51 \%)+20(20 \%)+10(14 \%)$ & ${ }^{2} \mathrm{~F}_{7 / 2}$ & 513322.0 & $510921.0(2401)$ & $\mathrm{T}$ \\
\hline 19 & $3 s^{2} 3 p^{4} 3 d$ & $(46 \%)+29(21 \%)+17(16 \%)$ & ${ }^{2} \mathrm{D}_{5 / 2}$ & 517550.0 & $513520.0(4030)$ & $\mathrm{T}$ \\
\hline 20 & $3 s^{2} 3 p^{4} 3 d$ & $(69 \%)+18(19 \%)$ & ${ }^{2} \mathrm{G}_{7 / 2}$ & 527000.0 & $526763.0(237)$ & \\
\hline 21 & $3 s^{2} 3 p^{4} 3 d$ & $(91 \%)$ & ${ }^{2} \mathrm{G}_{9 / 2}$ & 527270.0 & $528177.0(-907)$ & $\mathrm{T}$ \\
\hline 22 & $3 s^{2} 3 p^{4} 3 d$ & $(73 \%)+23(17 \%)$ & ${ }^{2} \mathrm{~F}_{5 / 2}$ & 528370.0 & $529036.0(-666)$ & $\mathrm{T}$ \\
\hline 23 & $3 s^{2} 3 p^{4} 3 d$ & $(78 \%)+22(14 \%)$ & ${ }^{2} \mathrm{~F}_{5 / 2}$ & - & 558016.0 & \\
\hline 24 & $3 s^{2} 3 p^{4} 3 d$ & $(79 \%)+18(15 \%)$ & ${ }^{2} \mathrm{~F}_{7 / 2}$ & 567200.0 & $564871.0(2329)$ & $\mathrm{T}$ \\
\hline 25 & $3 s^{2} 3 p^{4} 3 d$ & $(66 \%)+13(24 \%)$ & ${ }^{2} \mathrm{D}_{3 / 2}$ & - & 594911.0 & \\
\hline 26 & $3 s^{2} 3 p^{4} 3 d$ & $(73 \%)+19(15 \%)$ & ${ }^{2} \mathrm{D}_{5 / 2}$ & - & 603754.0 & \\
\hline 27 & $3 s^{2} 3 p^{4} 3 d$ & $(73 \%)+3(\mathrm{c} 220 \%)$ & ${ }^{2} \mathrm{~S}_{1 / 2}$ & 622836.0 & $622917.0(-81)$ & \\
\hline 28 & $3 s^{2} 3 p^{4} 3 d$ & $(48 \%)+11(40 \%)$ & ${ }^{2} \mathrm{P}_{3 / 2}$ & 648630.0 & $648743.0(-113)$ & \\
\hline 29 & $3 s^{2} 3 p^{4} 3 d$ & $(66 \%)+19(19 \%)+26(10 \%)$ & ${ }^{2} \mathrm{D}_{5 / 2}$ & 657240.0 & $656530.0(710)$ & \\
\hline 30 & $3 s^{2} 3 p^{4} 3 d$ & $(50 \%)+8(45 \%)$ & ${ }^{2} \mathrm{P}_{1 / 2}$ & 657360.0 & $658765.0(-1405)$ & \\
\hline 31 & $3 s^{2} 3 p^{4} 3 d$ & $(59 \%)+13(14 \%)+25(15 \%)$ & ${ }^{2} \mathrm{D}_{3 / 2}$ & 678520.0 & $678384.0(136)$ & $\mathrm{T}$ \\
\hline 242 & $3 s^{2} 3 p^{4} 4 s$ & $(89 \%)$ & ${ }^{4} \mathrm{P}_{5 / 2}$ & - & 1340554.0 & \\
\hline 246 & $3 s^{2} 3 p^{4} 4 s$ & $(79 \%)+262(13 \%)$ & ${ }^{4} \mathrm{P}_{3 / 2}$ & - & 1352730.0 & \\
\hline 262 & $3 s^{2} 3 p^{4} 4 s$ & $(68 \%)+246(14 \%)$ & ${ }^{2} \mathrm{P}_{3 / 2}$ & 1374200.0 & $1372231.0(1969)$ & \\
\hline 273 & $3 s^{2} 3 p^{4} 4 s$ & $(91 \%)$ & ${ }^{2} \mathrm{P}_{1 / 2}$ & 1385600.0 & $1385929.0(-329)$ & \\
\hline 282 & $3 s^{2} 3 p^{4} 4 s$ & $(91 \%)$ & ${ }^{2} \mathrm{D}_{5 / 2}$ & 1401000.0 & $1400752.0(248)$ & \\
\hline 283 & $3 s^{2} 3 p^{4} 4 s$ & $(85 \%)$ & ${ }^{2} \mathrm{D}_{3 / 2}$ & 1401600.0 & $1402352.0(-752)$ & \\
\hline 435 & $3 s^{2} 3 p^{4} 4 d$ & $(63 \%)+444(13 \%)$ & ${ }^{2} \mathrm{D}_{5 / 2}$ & 1666100.0 & $1664834.0(1266)$ & \\
\hline 462 & $3 s 3 p^{5} 4 s$ & $(72 \%)+709(\mathrm{c} 1411 \%)$ & ${ }^{2} \mathrm{P}_{3 / 2}$ & - & 1717741.0 & \\
\hline 477 & $3 s^{2} 3 p^{4} 4 f$ & $(80 \%)+491(12 \%)$ & ${ }^{4} \mathrm{~F}_{9 / 2}$ & 1797400.0 & $1795772.0(1628)$ & \\
\hline 483 & $3 s^{2} 3 p^{4} 4 f$ & $(90 \%)$ & ${ }^{4} \mathrm{G}_{11 / 2}$ & 1808000.0 & $1804379.0(3621)$ & \\
\hline 491 & $3 s^{2} 3 p^{4} 4 f$ & $(48 \%)+505(37 \%)$ & ${ }^{4} \mathrm{G}_{9 / 2}$ & 1811400.0 & $1810424.0(976)$ & \\
\hline 535 & $3 s^{2} 3 p^{4} 4 f$ & $(89 \%)$ & ${ }^{2} \mathrm{H}_{11 / 2}$ & 1848400.0 & $1849626.0(-1226)$ & \\
\hline
\end{tabular}

Notes. The experimental level energies $E_{\text {exp }}$ (Kaysers) are shown, together with those obtained from our scattering target and TEC $E_{\text {TEC }}$. Values in brackets indicate differences with the experimental energies. $T$ in the last column indicates a tentative experimental energy.

values, then re-run AUTOSTRUCTURE to obtain the target energies $E_{\mathrm{TEC}}$, with the TEC applied. The $E_{\mathrm{TEC}}$ and $E_{\mathrm{exp}}$ energies were used to calculate the radiative data.

We note that after having run the scattering calculations we have calculated the intensities of the strongest lines and then tentatively identified several levels, as we describe below. These tentative new energies are also listed in Table 1, with a "T" in the last column. We have not refined the TEC and re-run the calculations because of the uncertainty in the identifications of the lines and the corresponding level energies.

\section{R-matrix scattering calculation}

The R-matrix method used in the scattering calculation is described in Hummer et al. (1993) and Berrington et al. (1995). We performed the calculation in the inner region in LS coupling and included mass and Darwin relativistic energy corrections. The outer region calculation used the intermediate-coupling frame transformation method (ICFT) described by Griffin et al. (1998).

For the close-coupling expansion, we have retained the lowest 312 LS terms. This represents a significant improvement over the 14 LS target states included by Matthews et al. (1998), and an improvement over the $218 \mathrm{LS}$ terms we included for Fe X (Del Zanna et al. 2012). We recall that Matthews et al. (1998) adopted the LS R-matrix + JAJOM programs.

Given that our CC expansion is much smaller than the CI expansion, the highest levels in the $\mathrm{CC}$ expansion do not have reliable collision strengths, mostly because of mixing with other levels not included in the CC expansion. We have calculated the collision strengths of the fine-structure levels associated with the $312 \mathrm{LS}$ terms, but we only provide those associated with the lowest 589 levels, which include all the spectroscopically important levels (the No. 589 is the last $3 \mathrm{~s}^{2} 3 \mathrm{p}^{4} 4 \mathrm{f}$ level). 
Table 2. Collision strengths for a selection of transitions.

\begin{tabular}{|c|c|c|c|c|}
\hline$i-j$ & 20.1 & 39.9 & 59.8 & 80.2 \\
\hline $1-2$ & 0.16 & 0.15 & 0.15 & $\overline{0.15}$ \\
\hline $1-3$ & 0.60 & 0.70 & 0.76 & 0.81 \\
\hline $1-4$ & $3.7 \times 10^{-2}$ & $1.9 \times 10^{-2}$ & $1.2 \times 10^{-2}$ & $8.7 \times 10^{-3}$ \\
\hline $1-5$ & $5.2 \times 10^{-2}$ & $2.6 \times 10^{-2}$ & $1.5 \times 10^{-2}$ & $9.8 \times 10^{-3}$ \\
\hline $1-6$ & $2.3 \times 10^{-2}$ & $1.2 \times 10^{-2}$ & $8.3 \times 10^{-3}$ & $6.4 \times 10^{-3}$ \\
\hline $1-7$ & $9.4 \times 10^{-3}$ & $5.0 \times 10^{-3}$ & $3.2 \times 10^{-3}$ & $2.4 \times 10^{-3}$ \\
\hline $1-8$ & $9.6 \times 10^{-3}$ & $5.4 \times 10^{-3}$ & $3.7 \times 10^{-3}$ & $2.9 \times 10^{-3}$ \\
\hline $1-9$ & $3.6 \times 10^{-2}$ & $1.9 \times 10^{-2}$ & $1.3 \times 10^{-2}$ & $9.8 \times 10^{-3}$ \\
\hline $1-10$ & $3.0 \times 10^{-2}$ & $2.0 \times 10^{-2}$ & $1.7 \times 10^{-2}$ & $1.6 \times 10^{-2}$ \\
\hline $1-11$ & $3.6 \times 10^{-2}$ & $3.3 \times 10^{-2}$ & $3.3 \times 10^{-2}$ & $3.4 \times 10^{-2}$ \\
\hline $1-12$ & $2.0 \times 10^{-2}$ & $2.2 \times 10^{-2}$ & $2.4 \times 10^{-2}$ & $2.6 \times 10^{-2}$ \\
\hline $1-13$ & $3.2 \times 10^{-2}$ & $2.8 \times 10^{-2}$ & $2.8 \times 10^{-2}$ & $2.9 \times 10^{-2}$ \\
\hline $1-14$ & $2.0 \times 10^{-2}$ & $1.5 \times 10^{-2}$ & $1.4 \times 10^{-2}$ & $1.4 \times 10^{-2}$ \\
\hline $1-15$ & $2.3 \times 10^{-2}$ & $2.2 \times 10^{-2}$ & $2.3 \times 10^{-2}$ & $2.5 \times 10^{-2}$ \\
\hline $1-16$ & $1.4 \times 10^{-2}$ & $1.0 \times 10^{-2}$ & $9.2 \times 10^{-3}$ & $8.7 \times 10^{-3}$ \\
\hline $1-17$ & $3.8 \times 10^{-2}$ & $3.0 \times 10^{-2}$ & $2.8 \times 10^{-2}$ & $2.8 \times 10^{-2}$ \\
\hline $1-18$ & $4.4 \times 10^{-2}$ & $4.2 \times 10^{-2}$ & $4.3 \times 10^{-2}$ & $4.4 \times 10^{-2}$ \\
\hline $1-19$ & $3.7 \times 10^{-2}$ & $3.9 \times 10^{-2}$ & $4.1 \times 10^{-2}$ & $4.3 \times 10^{-2}$ \\
\hline $1-20$ & $1.7 \times 10^{-2}$ & $8.5 \times 10^{-3}$ & $4.9 \times 10^{-3}$ & $3.1 \times 10^{-3}$ \\
\hline $1-21$ & $5.0 \times 10^{-2}$ & $4.9 \times 10^{-2}$ & $5.1 \times 10^{-2}$ & $5.3 \times 10^{-2}$ \\
\hline $1-22$ & $2.1 \times 10^{-2}$ & $1.9 \times 10^{-2}$ & $1.9 \times 10^{-2}$ & $1.9 \times 10^{-2}$ \\
\hline $1-23$ & $4.0 \times 10^{-2}$ & $4.3 \times 10^{-2}$ & $4.8 \times 10^{-2}$ & $5.2 \times 10^{-2}$ \\
\hline $1-24$ & $3.2 \times 10^{-2}$ & $3.3 \times 10^{-2}$ & $10^{-2}$ & $3.7 \times 10^{-2}$ \\
\hline $1-25$ & $3.7 \times 10^{-2}$ & $4.3 \times 10^{-2}$ & $4.8 \times 10^{-2}$ & $5.3 \times 10^{-2}$ \\
\hline $1-26$ & $6.9 \times 10^{-3}$ & $3.9 \times 10^{-3}$ & $2.8 \times 10^{-3}$ & $2.4 \times 10^{-3}$ \\
\hline $1-27$ & 1.9 & 2.3 & 2.6 & 2.9 \\
\hline $1-28$ & 3.6 & 4.5 & 5.2 & 5.7 \\
\hline $1-29$ & 6.2 & 7.8 & 8.9 & 9.8 \\
\hline $1-30$ & 0.36 & 0.45 & 0.52 & 0.57 \\
\hline $1-31$ & $9.7 \times 10^{-2}$ & 0.12 & 0.13 & 0.14 \\
\hline $1-242$ & $9.5 \times 10^{-3}$ & $5.9 \times 10^{-3}$ & $5.5 \times 10^{-3}$ & $5.8 \times 10^{-3}$ \\
\hline $1-246$ & $1.2 \times 10^{-2}$ & $2.0 \times 10^{-2}$ & $2.8 \times 10^{-2}$ & $3.4 \times 10^{-2}$ \\
\hline $1-262$ & $4.1 \times 10^{-2}$ & $8.7 \times 10^{-2}$ & 0.12 & 0.15 \\
\hline $1-273$ & $1.4 \times 10^{-2}$ & $2.8 \times 10^{-2}$ & $4.0 \times 10^{-2}$ & $5.0 \times 10^{-2}$ \\
\hline $1-282$ & $3.2 \times 10^{-2}$ & $6.0 \times 10^{-2}$ & $8.5 \times 10^{-2}$ & 0.11 \\
\hline $1-283$ & $4.2 \times 10^{-3}$ & $2.2 \times 10^{-3}$ & $1.7 \times 10^{-3}$ & $1.6 \times 10^{-3}$ \\
\hline $1-435$ & $5.6 \times 10^{-2}$ & 0.12 & 0.18 & 0.23 \\
\hline $1-462$ & 0.33 & 0.37 & 0.39 & 0.39 \\
\hline $1-477$ & $2.8 \times 10^{-2}$ & $9.2 \times 10^{-3}$ & $4.4 \times 10^{-3}$ & $2.5 \times 10^{-3}$ \\
\hline $1-483$ & $1.8 \times 10^{-2}$ & $6.3 \times 10^{-3}$ & $3.6 \times 10^{-3}$ & $2.6 \times 10^{-3}$ \\
\hline $1-491$ & $1.5 \times 10^{-2}$ & $1.0 \times 10^{-2}$ & $1.0 \times 10^{-2}$ & $1.0 \times 10^{-2}$ \\
\hline $1-535$ & $1.3 \times 10^{-2}$ & $1.2 \times 10^{-2}$ & $1.4 \times 10^{-2}$ & $1.5 \times 10^{-2}$ \\
\hline
\end{tabular}

Notes. The collision strengths are shown for four values of incident electron energies (Rydbergs) above thresholds.

The expansion of each scattered electron partial wave was done over a basis of 22 functions within the R-matrix boundary and the partial wave expansion extended to a maximum total orbital angular momentum quantum number of $L=16$. This produced accurate collision strengths up to about 80 Ryd.

The outer region calculation includes exchange up to a total angular momentum quantum number $J=26 / 2$. We have supplemented the exchange contributions with a non-exchange calculation extending from $J=28 / 2$ to $J=74 / 2$. Collision strengths were "topped-up" to infinite partial waves following Burgess (1974), Badnell \& Griffin (2001).

The resonance region was calculated with 5400 points. A coarse energy mesh was chosen above all resonances.

To improve the collision strengths of the strongly mixed levels, we have included the TEC into the ICFT method as described in Del Zanna \& Badnell (2014). This method can affect some decays from highly mixed levels. Table 2 lists a selection
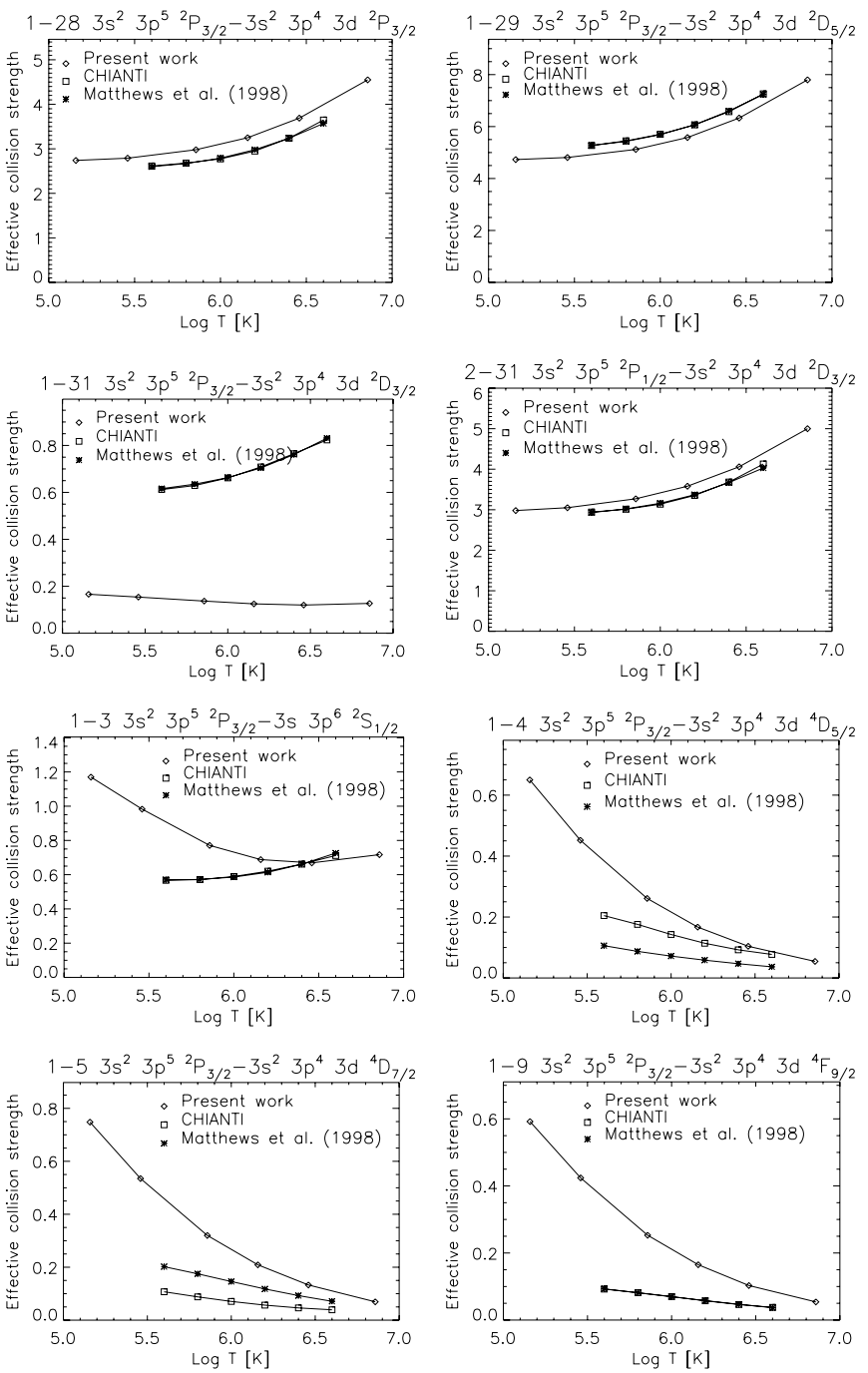

Fig. 1. Thermally-averaged collision strengths for a selection of transitions compared to those calculated by Matthews et al. (1998) and those tabulated in the CHIANTI database.

of collision strengths for the main transitions, for incident electron energies above thresholds, where resonance enhancements are not present.

Finally, the collision strengths were extended to high energies by interpolation using the appropriate high-energy limits in the Burgess \& Tully (1992) scaled domain. The high-energy limits were calculated with AUTOSTRUCTURE (see Burgess et al. 1997; Chidichimo et al. 2003), including the TEC.

The temperature-dependent effective collisions strengths $\Upsilon(i-j)$ were calculated by assuming a Maxwellian electron distribution and linear integration with the final energy of the colliding electron. Figure 1 shows a few examples, compared to those calculated by Matthews et al. (1998) and those tabulated in the CHIANTI database. We only show a few collision strengths that are populating some of the most important levels, as described in the next Section. The plots in Fig. 1 show significant differences with those calculated by Matthews et al. (1998).

Some of the large differences in the thermally-averaged collision strengths are mostly present towards low temperatures, due to the resonance enhancements caused by our much larger CC expansion. Some examples are provided in the figure, the transitions to the $3 \mathrm{~s} 3 \mathrm{p}^{6}{ }^{2} \mathrm{~S}_{1 / 2}, 3 \mathrm{~s}^{2} 3 \mathrm{p}^{4} 3 \mathrm{~d}^{4} \mathrm{D}_{5 / 2,7 / 2}$, and $3 s^{2} 3 p^{4} 3 d{ }^{4} F_{9 / 2}$ levels. In these three cases we note 
Table 3. A selection of the strongest Ni XII lines.

\begin{tabular}{|c|c|c|c|c|c|c|c|}
\hline$i-j$ & Levels & Int & $g f$ & $A_{j i}\left(\mathrm{~s}^{-1}\right)$ & $\lambda_{\exp }(\AA)$ & $\lambda_{\text {th }}(\AA)$ & \\
\hline $1-29$ & $3 s^{2} 3 p^{5}{ }^{2} P_{3 / 2}-3 s^{2} 3 p^{4} 3 d^{2} D_{5 / 2}$ & 1.0 & 4.35 & $2.1 \times 10^{11}$ & 152.151 & 152.32 & \\
\hline $1-28$ & $3 s^{2} 3 p^{5}{ }^{2} P_{3 / 2}-3 s^{2} 3 p^{4} 3 d^{2} P_{3 / 2}$ & $0.58(0.49)$ & 2.50 & $1.8 \times 10^{11}$ & 154.171 & 154.14 & \\
\hline $1-27$ & $3 s^{2} 3 p^{5}{ }^{2} P_{3 / 2}-3 s^{2} 3 p^{4} 3 d^{2} S_{1 / 2}$ & $0.25(0.21)$ & 1.17 & $1.5 \times 10^{11}$ & 160.556 & 160.54 & \\
\hline $1-5$ & $3 s^{2} 3 p^{5}{ }^{2} P_{3 / 2}-3 s^{2} 3 p^{4} 3 d^{4} D_{7 / 2}$ & $0.20(0.06)$ & - & 91. & 220.247 & 221.87 & $\mathrm{~T}$ \\
\hline $1-3$ & $3 s^{2} 3 p^{5}{ }^{2} P_{3 / 2}-3 s 3 p^{6}{ }^{2} S_{1 / 2}$ & $0.14(0.09)$ & 0.13 & $5.1 \times 10^{9}$ & 295.321 & 295.10 & \\
\hline $1-4$ & $3 s^{2} 3 p^{5}{ }^{2} P_{3 / 2}-3 s^{2} 3 p^{4} 3 d^{4} D_{5 / 2}$ & $6.6(2.4) \times 10^{-2}$ & $3.7 \times 10^{-4}$ & $8.3 \times 10^{6}$ & 220.870 & 222.40 & $\mathrm{~T}$ \\
\hline $2-27$ & $3 s^{2} 3 p^{5}{ }^{2} P_{1 / 2}-3 s^{2} 3 p^{4} 3 d^{2} S_{1 / 2}$ & $6.2(6.0) \times 10^{-2}$ & 0.31 & $3.7 \times 10^{10}$ & 166.887 & 167.09 & \\
\hline $2-30$ & $3 s^{2} 3 p^{5}{ }^{2} \mathrm{P}_{1 / 2}-3 s^{2} 3 p^{4} 3 d^{2} \mathrm{P}_{1 / 2}$ & $5.6(8.2) \times 10^{-2}$ & 1.00 & $1.3 \times 10^{11}$ & 157.796 & 157.65 & \\
\hline $1-19$ & $3 s^{2} 3 p^{5}{ }^{2} P_{3 / 2}-3 s^{2} 3 p^{4} 3 d^{2} D_{5 / 2}$ & $4.8(1.3) \times 10^{-2}$ & $6.1 \times 10^{-3}$ & $1.8 \times 10^{8}$ & - & 194.73 & \\
\hline $1-17$ & $3 s^{2} 3 p^{5}{ }^{2} \mathrm{P}_{3 / 2}-3 s^{2} 3 p^{4} 3 d^{4} \mathrm{P}_{5 / 2}$ & $4.3(0.6) \times 10^{-2}$ & $4.6 \times 10^{-3}$ & $1.3 \times 10^{8}$ & - & 196.56 & \\
\hline $1-22$ & $3 s^{2} 3 p^{5}{ }^{2} P_{3 / 2}-3 s^{2} 3 p^{4} 3 d^{2} F_{5 / 2}$ & $3.9(0.7) \times 10^{-2}$ & $9.5 \times 10^{-4}$ & $3.0 \times 10^{7}$ & - & 189.02 & \\
\hline $2-31$ & $3 s^{2} 3 p^{5}{ }^{2} P_{1 / 2}-3 s^{2} 3 p^{4} 3 d^{2} D_{3 / 2}$ & $4.0(13) \times 10^{-2}$ & 2.79 & $2.0 \times 10^{11}$ & 152.697 & 152.92 & $\mathrm{~T}$ \\
\hline $2-3$ & $3 s^{2} 3 p^{5}{ }^{2} P_{1 / 2}-3 s 3 p^{6}{ }^{2} S_{1 / 2}$ & $6.1(4.0) \times 10^{-2}$ & $6.5 \times 10^{-2}$ & $2.2 \times 10^{9}$ & 317.474 & 318.04 & \\
\hline $1-2$ & $3 s^{2} 3 p^{5}{ }^{2} \mathrm{P}_{3 / 2}-3 s^{2} 3 p^{5}{ }^{2} \mathrm{P}_{1 / 2}$ & $0.57(0.65)$ & - & $2.6 \times 10^{2}$ & 4232.09 & 4091.00 & \\
\hline $5-9$ & $3 s^{2} 3 p^{4} 3 d^{4} D_{7 / 2}-3 s^{2} 3 p^{4} 3 d^{4} F_{9 / 2}$ & $9.4(3.4) \times 10^{-2}$ & - & 28. & 3171.18 & 3014.08 & \\
\hline $9-21$ & $3 s^{2} 3 p^{4} 3 d^{4} F_{9 / 2}-3 s^{2} 3 p^{4} 3 d^{2} G_{9 / 2}$ & $3.8(1.4) \times 10^{-2}$ & - & $2.0 \times 10^{2}$ & 2398.08 & 2258.57 & \\
\hline $5-18$ & $3 s^{2} 3 p^{4} 3 d^{4} D_{7 / 2}-3 s^{2} 3 p^{4} 3 d^{2} F_{7 / 2}$ & $2.5(0.47) \times 10^{-2}$ & - & 90. & 1686.74 & 1661.20 & \\
\hline $5-10$ & $3 s^{2} 3 p^{4} 3 d^{4} D_{7 / 2}-3 s^{2} 3 p^{4} 3 d^{4} F_{7 / 2}$ & $1.9(0.66) \times 10^{-2}$ & - & 18. & 2580.38 & 2492.77 & \\
\hline $9-18$ & $3 s^{2} 3 p^{4} 3 d^{4} F_{9 / 2}-3 s^{2} 3 p^{4} 3 d^{2} F_{7 / 2}$ & $1.8(0.30) \times 10^{-2}$ & - & 57. & 3603.34 & 3701.00 & \\
\hline $10-20$ & $3 s^{2} 3 p^{4} 3 d^{4} F_{7 / 2}-3 s^{2} 3 p^{4} 3 d^{2} G_{7 / 2}$ & $1.3(0.28) \times 10^{-2}$ & - & 84. & 2923.12 & 2783.65 & \\
\hline $9-24$ & $3 s^{2} 3 p^{4} 3 d^{4} F_{9 / 2}-3 s^{2} 3 p^{4} 3 d^{2} F_{7 / 2}$ & $1.1(0.37) \times 10^{-2}$ & - & $2.3 \times 10^{2}$ & 1225.05 & 1235.02 & \\
\hline $3-462$ & $3 s 3 p^{6}{ }^{2} S_{1 / 2}-3 s 3 p^{5} 4 s^{2} P_{3 / 2}$ & $1.9 \times 10^{-2}$ & 0.25 & $8.0 \times 10^{10}$ & - & 72.52 & \\
\hline $1-282$ & $3 s^{2} 3 p^{5}{ }^{2} P_{3 / 2}-3 s^{2} 3 p^{4} 4 s^{2} D_{5 / 2}$ & $1.2 \times 10^{-2}$ & 0.30 & $6.7 \times 10^{10}$ & 71.378 & 71.39 & \\
\hline $1-262$ & $3 s^{2} 3 p^{5}{ }^{2} \mathrm{P}_{3 / 2}-3 s^{2} 3 p^{4} 4 s^{2} \mathrm{P}_{3 / 2}$ & $1.1 \times 10^{-2}$ & 0.45 & $1.4 \times 10^{11}$ & 72.770 & 72.87 & \\
\hline $1-435$ & $3 s^{2} 3 p^{5}{ }^{2} P_{3 / 2}-3 s^{2} 3 p^{4} 4 d^{2} D_{5 / 2}$ & $5.8 \times 10^{-3}$ & 1.00 & $3.1 \times 10^{11}$ & 60.020 & 60.07 & \\
\hline $1-246$ & $3 s^{2} 3 p^{5}{ }^{2} P_{3 / 2}-3 s^{2} 3 p^{4} 4 s^{4} P_{3 / 2}$ & $4.5 \times 10^{-3}$ & 0.10 & $3.0 \times 10^{10}$ & - & 73.93 & \\
\hline $1-242$ & $3 s^{2} 3 p^{5}{ }^{2} P_{3 / 2}-3 s^{2} 3 p^{4} 4 s^{4} P_{5 / 2}$ & $3.7 \times 10^{-3}$ & $1.5 \times 10^{-2}$ & $2.9 \times 10^{9}$ & - & 74.60 & \\
\hline $22-526$ & $3 s^{2} 3 p^{4} 3 d^{2} F_{5 / 2}-3 s^{2} 3 p^{4} 4 f^{2} G_{7 / 2}$ & $3.1 \times 10^{-3}$ & 2.33 & $3.3 \times 10^{11}$ & - & 76.38 & \\
\hline $2-283$ & $3 s^{2} 3 p^{5}{ }^{2} P_{1 / 2}-3 s^{2} 3 p^{4} 4 s^{2} D_{3 / 2}$ & $2.4 \times 10^{-3}$ & 0.27 & $8.6 \times 10^{10}$ & 72.570 & 72.57 & \\
\hline $2-273$ & $3 s^{2} 3 p^{5}{ }^{2} \mathrm{P}_{1 / 2}-3 s^{2} 3 p^{4} 4 s^{2} \mathrm{P}_{1 / 2}$ & $2.4 \times 10^{-3}$ & 0.17 & $1.0 \times 10^{11}$ & 73.423 & 73.45 & \\
\hline $9-483$ & $3 s^{2} 3 p^{4} 3 d^{4} F_{9 / 2}-3 s^{2} 3 p^{4} 4 f^{4} G_{11 / 2}$ & $2.3 \times 10^{-3}$ & 6.47 & $6.3 \times 10^{11}$ & 75.618 & 75.73 & \\
\hline $1-273$ & $3 s^{2} 3 p^{5}{ }^{2} \mathrm{P}_{3 / 2}-3 s^{2} 3 p^{4} 4 s^{2} \mathrm{P}_{1 / 2}$ & $2.1 \times 10^{-3}$ & 0.15 & $9.3 \times 10^{10}$ & 72.171 & 72.15 & \\
\hline $14-498$ & $3 s^{2} 3 p^{4} 3 d^{4} F_{5 / 2}-3 s^{2} 3 p^{4} 4 f^{4} G_{7 / 2}$ & $2.1 \times 10^{-3}$ & 2.33 & $3.4 \times 10^{11}$ & - & 75.78 & \\
\hline $5-477$ & $3 s^{2} 3 p^{4} 3 d^{4} D_{7 / 2}-3 s^{2} 3 p^{4} 4 f^{4} F_{9 / 2}$ & $1.9 \times 10^{-3}$ & 4.44 & $5.4 \times 10^{11}$ & 74.440 & 74.35 & \\
\hline
\end{tabular}

Notes. Column 3 shows the relative intensities (photons) $I=N_{j} A_{j i} / N_{\mathrm{e}}$ of the strongest lines, relative to the main transition. The intensities were calculated at an electron density of $10^{9} \mathrm{~cm}^{-3}$ and $\log T[\mathrm{~K}]=6.25$, the temperature of peak ion abundance in equilibrium. Values in brackets are those calculated with the CHIANTI database. Columns 4 and 5 show the $g f$ and $A$ values calculated in this work with TECs. The $\lambda_{\text {exp }}(\AA)$ are our experimental wavelengths (in vacuum), while the $\lambda_{\text {th }}(\AA)$ values are our theoretical values obtained with the TECs. A "T" in the last column indicates a tentative new experimental wavelength.

a discrepancy between the values published by Matthews et al. (1998) and the interpolated values in the CHIANTI database.

\section{Comparison with observations}

We have calculated the level populations using our collisions strengths and $A$-values. Proton excitation as available in the CHIANTI database v.8 (Del Zanna et al. 2015) has been included. The intensities of the strongest lines are shown in Table 3, for an electron density of $10^{9} \mathrm{~cm}^{-3}$, typical of a solar active region, and the temperature of peak ion abundance in equilibrium. This table also shows in brackets the intensities as calculated with the CHIANTI database. There is relatively good agreement for the three strongest transitions; however, significant differences are found for all the other lines, in particular for the decays of the $3 \mathrm{~s}^{2} 3 \mathrm{p}^{4} 3 \mathrm{~d}^{4} \mathrm{D}_{7 / 2,5 / 2}$. There are also significant enhancements in the forbidden lines.
We find good agreement between the predicted and observed intensities of the three main soft X-ray lines observed by Malinovsky \& Heroux (1973) on the Sun, as shown in Fig. 2 (bottom). This figure shows the "emissivity ratio" curves

$F_{j i}=\frac{I_{\mathrm{ob}} N_{\mathrm{e}}}{N_{j}\left(N_{\mathrm{e}}, T_{\mathrm{e}}\right) A_{j i}} C$

for each line as a function of the electron density $N_{\mathrm{e}} . I_{\mathrm{ob}}$ is the observed intensity of the line (photon units), $N_{j}\left(N_{\mathrm{e}}, T_{\mathrm{e}}\right)$ is the population of the upper level $j$ relative to the total number density of the ion, calculated at a fixed electron temperature $T_{\mathrm{e}}=1.8 \mathrm{MK}$, the temperature of peak ion abundance assuming ionization equilibrium. $A_{j i}$ is the spontaneous radiative transition probability, and $C$ is a scaling constant chosen so the emissivity ratio is near unity. If agreement between experimental and theoretical intensities is present, all lines should be closely spaced. The crossing of the curves gives the electron density. 


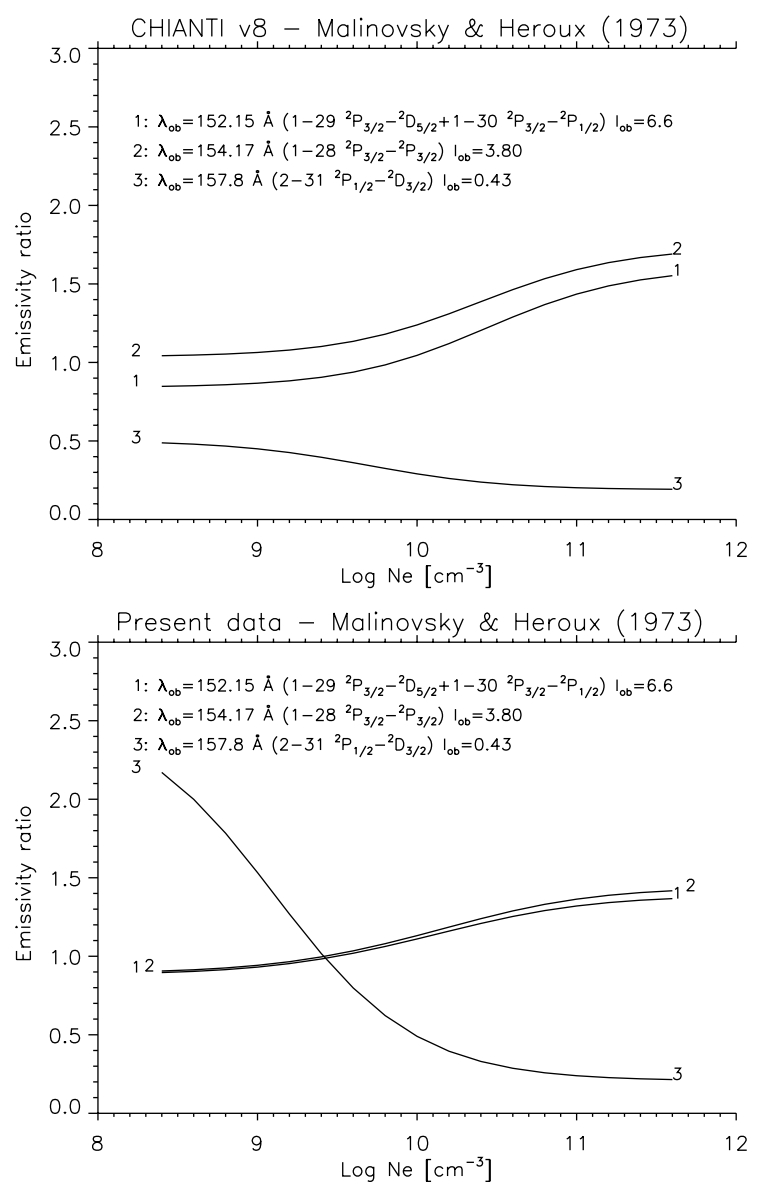

Fig. 2. Emissivity ratio curves relative to the soft X-ray solar Ni XII observed by Malinovsky \& Heroux (1973). The observed intensities $I_{\mathrm{ob}}$ are in ergs. The top plot is obtained with CHIANTI v.8, while the lower one with the present atomic data.

The CHIANTI ion model has a small 20\% discrepancy (see Fig. 2, top) in the ratio of the two strongest lines at 152.15 and $154.17 \AA$, decays from the $3 \mathrm{~s}^{2} 3 \mathrm{p}^{4} 3 \mathrm{~d}^{2} \mathrm{D}_{5 / 2}$ (No. 29) and $3 \mathrm{~s}^{2} 3 \mathrm{p}^{4}$ $3 \mathrm{~d}^{2} \mathrm{P}_{3 / 2}$ (No. 28) levels. The collision strengths of the allowed transitions to these levels are indeed similar to those calculated by Matthews et al. (1998; see top left plot of Fig. 1).

The 2-31 line is clearly blended in the Malinovsky \& Heroux (1973) spectrum, and indeed the authors indicate that. The actual intensity of this line is likely to be lower, which would lower the emissivity curve of this line and lower the density. We note that the electron density obtained from the Fe XIV lines, which are formed over similar temperatures as Ni XII, is about $10^{9} \mathrm{~cm}^{-3}$.

On the other hand, the CHIANTI ion model is inconsistent with the observations, as shown in Fig. 2 (top). The observed intensity of the 2-31 line would have to be twice the reported value. There is also a clear problem in the density sensitivity of the 2-31 line as calculated with the CHIANTI ion model. The upper level No. $31\left(3 \mathrm{~s}^{2} 3 \mathrm{p}^{4} 3 \mathrm{~d}^{2} \mathrm{D}_{3 / 2}\right)$ is populated (at the density and temperature of the table) by direct excitation from the ground state ${ }^{2} \mathrm{P}_{3 / 2}$ (49\%) and the ${ }^{2} \mathrm{P}_{1 / 2}(41 \%)$. As shown in Fig. 1, the stronger collision strengths from the ${ }^{2} \mathrm{P}_{1 / 2}$ are in relative good agreement with the values calculated by Matthews et al. (1998); however, the collision strengths from the ${ }^{2} \mathrm{P}_{3 / 2}$ are very much different. Our oscillator strength for the decay to the ground state does not vary much with the addition of the TEC, and is close to the value calculated by Fawcett (1987). Our
Table 4. Observed wavelengths of Ni XII lines.

\begin{tabular}{lrl}
\hline \hline$i-j$ & $\lambda_{\text {exp }}(\AA)$ & Ref. \\
\hline $1-2$ & $4231.2 \pm 0.1($ air $)$ & J71 \\
$2-3$ & $317.475 \pm 0.008$ & FH80 \\
$1-3$ & $295.321 \pm 0.008$ & FH80 \\
$1-4$ & $220.87 \pm 0.05$ & Be76 \\
$1-5$ & $220.247 \pm 0.05$ & Be76 \\
$5-9$ & $3167.0 \pm 0.1($ air $)$ & J71 \\
$1-14$ & $202.0 \pm 0.1$ & T93 \\
$1-16$ & $198.289 \pm 0.05$ & D12 \\
$5-18$ & $1686.74 \pm 0.02$ & S79 \\
$1-19$ & $193.218 \pm 0.05$ & D12 \\
$5-20$ & $1370.52 \pm 0.02$ & S77 \\
$1-22$ & $189.26 \pm 0.05$ & D12 \\
$9-24$ & $1225.05 \pm 0.02$ & S79 \\
$1-27$ & $160.556 \pm 0.05$ & S92 \\
$1-28$ & $154.171 \pm 0.05$ & S92 \\
$1-29$ & $152.151 \pm 0.05$ & S92 \\
$2-30$ & $157.795 \pm 0.05$ & S92 \\
$2-31$ & $152.697 \pm 0.05 ?$ & R79 \\
$1-262$ & $72.77 \pm 0.01$ & F72 \\
$1-273$ & $72.17 \pm 0.01$ & F72 \\
$1-282$ & $71.4($ blended) & F72 \\
$2-283$ & $72.57 \pm 0.01$ & F72 \\
$1-435$ & $60.02 \pm 0.01$ & F72 \\
$5-477$ & $74.44 \pm 0.01$ & F72 \\
$9-483$ & $75.62 \pm 0.01$ & F72 \\
$10-491$ & $75.83 \pm 0.01$ & F72 \\
$21-535$ & $75.69 \pm 0.01$ & F72 \\
\hline & &
\end{tabular}

References. S77: Sandlin et al. (1977); S79: Sandlin \& Tousey (1979); J71: Jefferies et al. (1971); FH80: Fawcett \& Hatter (1980); Be76: Behring et al. (1976); T93: Trabert et al. (1993); D12: Del Zanna (2012b); S92: Sugar et al. (1992); R79: Ryabtsev (1979); F72: Fawcett et al. (1972).

collision strengths tend to the high-energy limits, so there seems to be a problem in the Matthews et al. (1998) calculations.

We now briefly comment on some significant differences in the predicted intensities of other lines, shown in Table 3 . The $1-33 s^{2} 3 p^{5}{ }^{2} \mathrm{P}_{3 / 2}-3 \mathrm{~s} 3 \mathrm{p}^{6}{ }^{2} \mathrm{~S}_{1 / 2}$ transition is significantly enhanced. Only about $72 \%$ of the population of the upper level is due to direct excitation form the ground state, which is enhanced with the present calculation, as shown in Fig. 1. About $27 \%$ of its populations comes from cascading from higher levels which were not present in the previous atomic model. This is the reason for such a large difference, as we also found for Fe X (Del Zanna et al. 2012) and other coronal ions.

The $1-53 s^{2} 3 p^{5}{ }^{2} P_{3 / 2}-3 s^{2} 3 p^{4} 3 d^{4} D_{7 / 2}$ is also very much enhanced in our model. It turns out that only $15 \%$ of its population comes from direct excitation form the ground state, which is enhanced with the present calculation, as shown in Fig. 1. About $84 \%$ comes from upper levels, for example $39 \%$ from level No. $9,3 \mathrm{~s}^{2} 3 \mathrm{p}^{4} 3 \mathrm{~d}^{4} \mathrm{~F}_{9 / 2}$. In turn, this level No. 9 is populated by $15 \%$ from direct excitation form the ground state, which is enhanced with the present calculation, as shown in Fig. 1. The rest is due to cascading from higher levels, which are more populated because of increased collision strengths due to the larger number of resonances in our target.

\subsection{A discussion on level energies and line identifications}

We now briefly summarise the levels with known experimental energies. Table 4 lists the main wavelengths we have used 
to establish the experimental energies. The strongest Ni XII lines are produced by the $3 \mathrm{~s}^{2} 3 \mathrm{p}^{4} 3 \mathrm{~d}$ levels in the soft X-rays around $150 \AA$ A. The lines were identified by B.C. Fawcett (Gabriel et al. 1965, 1966; Fawcett \& Hayes 1972), using laboratory spectra. These lines have been observed with high and moderate spectral resolution by sounding rocket flights in the 1960's (Behring et al. 1972; Malinovsky \& Heroux 1973). We adopt for the strongest lines the wavelength measurements of Sugar et al. (1992). We note a mistake in Sugar et al. (1992), the line observed at $159.970 \AA$ is almost all due to Ni X, and not Ni XII. We also note that the wavelength of the $3 s^{2} 3 p^{5}{ }^{2} P_{1 / 2}-3 s^{2} 3 p^{4} 3 d{ }^{2} D_{3 / 2}(2-$ 31) line is still somewhat uncertain, as we discuss below.

The forbidden line within the ground configuration was observed by Jefferies et al. (1971) during the 1965 eclipse. The decays from the $3 \mathrm{~s} 3 \mathrm{p}^{6}{ }^{2} \mathrm{~S}_{1 / 2}$ level were identified by Fawcett $\&$ Hatter (1980).

Not all of the lower levels of the $3 \mathrm{~s}^{2} 3 \mathrm{p}^{4} 3 \mathrm{~d}$ configuration are known. Edlen \& Smitt (1978) suggested the identification of several forbidden lines observed in the UV and visible. This established the energies of several levels (Nos. 9, 10, 18, 20, 21, 24 , see Table 1) relative to the $3 \mathrm{~s}^{2} 3 \mathrm{p}^{4} 3 \mathrm{~d}^{4} \mathrm{D}_{7 / 2}^{\mathrm{e}}$ (level No. 5). The decay to the ground state from this ${ }^{4} \mathrm{D}_{7 / 2}^{\mathrm{e}}$ level is a relatively strong line with our new atomic data, so this line ought to be observable. We suggest that it is the line observed by Behring et al. (1976) at $220.247 \AA$. We base this suggestion on the differences between the observed and theoretical energy for this level that we obtained for Fe X, but also note that NIST suggests a similar energy. The theoretical splitting between the ${ }^{4} \mathrm{D}_{7 / 2}^{\mathrm{e}}$ level and the ${ }^{4} D_{5 / 2}^{\mathrm{e}}$ (level No. 4) agrees within 200 Kaysers with the decay from the ${ }^{4} \mathrm{D}_{5 / 2}^{\mathrm{e}}$ level being the line observed by Behring et al. (1976) at $220.87 \AA$.

Finally, a few decays from $3 s^{2} 3 p^{4} 4 s, 3 s^{2} 3 p^{4} 4 d, 3 s^{2} 3 p^{4} 4 f$ levels were identified by Fawcett et al. (1972) in the soft X-rays. As in the case of Fe X (Del Zanna et al. 2012), we predict that the decay from the $3 \mathrm{~s} 3 \mathrm{p}^{5} 4 \mathrm{~s}^{2} \mathrm{P}_{3 / 2}$ level to be stronger than the decays from the $3 \mathrm{~s}^{2} 3 \mathrm{p}^{4} 4 \mathrm{~s}$. In the case of $\mathrm{Fe} \mathrm{X}$ and the other coronal iron ions we were able to identify such soft X-ray transitions because they are relatively strong in solar spectra (Del Zanna 2012a), but in the case of nickel this is not simple.

\subsubsection{The wavelength of the $3 s^{2} 3 p^{5}$ ${ }^{2} P_{1 / 2}-3 s^{2} 3 p^{4} 3 d^{2} D_{3 / 2}$ line}

We note a puzzle regarding the wavelength of the $3 \mathrm{~s}^{2} 3 \mathrm{p}^{5}{ }^{2} \mathrm{P}_{1 / 2}-$ $3 \mathrm{~s}^{2} 3 \mathrm{p}^{4} 3 \mathrm{~d}^{2} \mathrm{D}_{3 / 2}(2-31)$ line. This line is weak at low densities, but becomes strong at high densities, as we have seen. Gabriel et al. (1965) identified this line with a laboratory line observed at $152.95 \AA$. The same identification is reported in Gabriel et al. (1966), Fawcett \& Hayes (1972). Fawcett \& Hayes (1972) actually report the observation of the decay to the ground state, at $147.60 \AA$.

Goldsmith \& Fraenkel (1970) used a vacuum spark and remeasured the nickel lines, providing a different identification for the 2-31 line, observed at $153.174 \AA$. The decay to the ground state was listed with zero intensity at $147.847 \AA$ (we note that the other lines had similar wavelengths to those measured by Fawcett).

However, the branching ratio of the two decays is 0.024 , i.e. the decay to the ground state is only $2.4 \%$ the intensity of the 2-31 line. So it is questionable that the decay to the ground

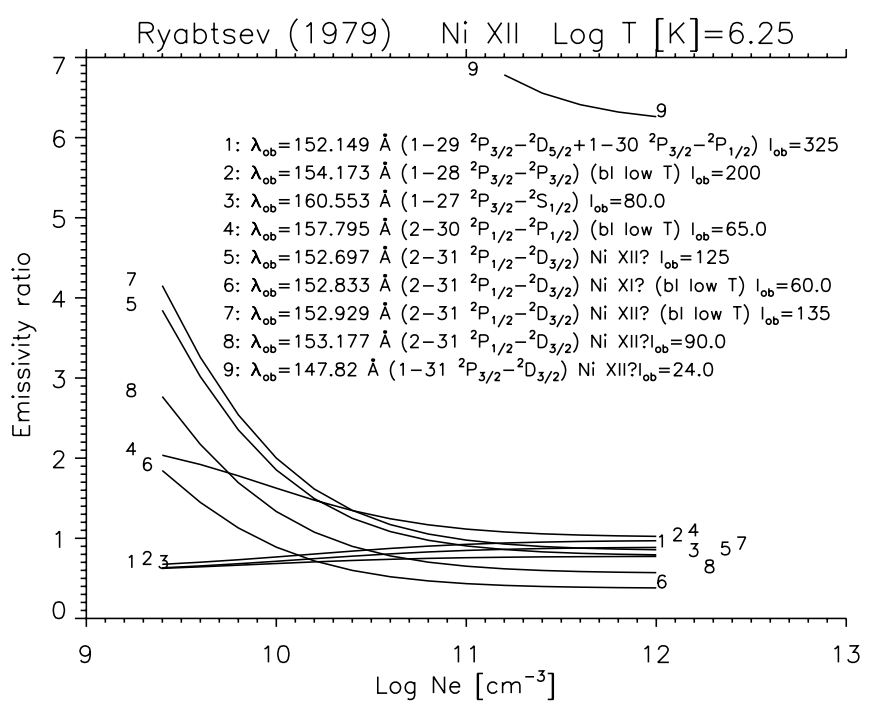

Fig. 3. Emissivity ratio curves relative to the soft X-ray Ni XII observed by Ryabtsev (1979).

state was actually observed by Fawcett or Goldsmith \& Fraenkel (1970). We return to this issue below.

Several laboratory spectra followed (see e.g. Wouters et al. 1988; Mattioli et al. 2004), but they did not have a sufficiently high spectral resolution to settle this issue. Mattioli et al. (2004) noted that the wavelength of the 2-31 line is consistent with the Fawcett measurement. Surprisingly, Sugar et al. (1992) does not list this transition. Keenan et al. (2000) reported observations with the Joint European Torus (JET) tokamak, and a wavelength of $152.90 \AA$ for this line. However, we note that the profile of this line in the JET spectra looks asymmetric and the spectrometer used had a medium resolution of $0.2 \AA$.

To help resolve the issue we have considered the excellent vacuum spark spectra obtained by Ryabtsev (1979). The spectrograph used for the measurements had a radius of $3 \mathrm{~m}$, so these spectra are the best in terms of spectral resolution. Lines from titanium were used as reference to establish the wavelength calibration. The Ryabtsev (1979) wavelengths of the strongest Ni XII lines are in agreement with those of Sugar et al. (1992) within a few $\mathrm{m} \AA$.

Ryabtsev (1979) report observations of nickel lines produced by a low-induction vacuum spark, with two settings. With the "cold" setting only low charge states of nickel were present. With the "hot" setting, higher ionization stages such as Ni XII were present. The lines that are known to pertain to lower charge-state ions such as Ni $\mathrm{X}$ have intensities that do not change much from the "cold" to the "hot" spectrum. Several of the Ni XII lines are clearly blended with lower charge-state ions, since they have considerable intensities in the "cold" spectrum. If we assume that no blending from higher ionization stages is present, and remove the lower charge-state contribution by subtracting the intensities of the "cold" spectrum from the "hot" spectrum, we obtain the emissivity ratio curves shown in Fig. 3. The agreement between predicted and observed intensities is truly remarkable, within only $20 \%$ at high densities, as we would expect in the vacuum spark spectra (the level populations reach Boltzmann equilibrium around $10^{11} \mathrm{~cm}^{-3}$ ).

Ryabtsev (1979) observed a line at $147.820 \AA$, which is listed as the same line observed by Goldsmith \& Fraenkel (1970) at $147.847 \AA$. As the Fig. 3 shows, this line cannot be the decay of the $3 s^{2} 3 p^{4} 3 d^{2} D_{3 / 2}$ to the ground state, having an intensity 
about a factor of six too high, as we anticipated. This means that the previous wavelenghts of the decay to the $3 \mathrm{~s}^{2} 3 \mathrm{p}^{5}{ }^{2} \mathrm{P}_{1 / 2}$ level (the 2-31 transition) based on the decay to the ground state should be treated with caution.

With regard to the 2-31 transition itself, Ryabtsev (1979) lists four nickel lines around $153 \AA$, at 152.697, 152.833, 152.929, and $153.177 \AA$. The line at $152.833 \AA$ is listed as a possible Ni XI line, because of its intensity in the cold and hot spectra. Its intensity is a factor of two too low compared to our prediction, so we discard it as a possible candidate. The line at $153.177 \AA$ is clearly the same line observed by Goldsmith \& Fraenkel (1970) at $153.174 \AA$. However, it is unlikely that this line is the 2-31 transition, because its observed intensity is also too low.

We are then left with two possibilities, the lines at 152.697 and $152.929 \AA$. These lines have similar intensities, and would be blended in most medium-resolution spectra, including the solar one of Malinovsky \& Heroux (1973), and the JET tokamak (Keenan et al. 2000; Mattioli et al. 2004). We have re-measured the wavelength of the line observed in the Malinovsky \& Heroux (1973) spectrum, obtaining a value of $152.80 \pm 0.10 \AA$, i.e. in between the two wavelengths of the candidate lines observed by Ryabtsev (1979).

However, the 152.697 and $152.929 \AA$ lines would be well separated in the high-resolution solar spectrum of Behring et al. (1972). These authors only list one unidentified line at $152.703 \AA$, a wavelength very close to $152.697 \AA$. We therefore suggest this new identification. We note that most of the lines listed by Ryabtsev (1979) would not be observable in the lowdensity solar corona, but the 2-31 transition should be, although the approximate intensity given by Behring et al. (1972) to the $152.703 \AA$ line is larger than what it should be.

Our suggestion is also supported by its predicted wavelength based on the theoretical splitting between the $3 s^{2} 3 p^{4} 3 d{ }^{2} D_{5 / 2,3 / 2}$ levels. The observed energy of the ${ }^{2} \mathrm{D}_{5 / 2}$ level is $710 \mathrm{~cm}^{-1}$ higher than the theoretical energy obtained with the TEC (cf. Table 1). If the $153.177 \AA$ line was the 2-31 transition, the energy of the ${ }^{2} \mathrm{D}_{3 / 2}$ level would be $1916 \mathrm{~cm}^{-1}$ lower than predicted. If the $152.929 \AA$ line was the 2-31 transition, the energy of the ${ }^{2} \mathrm{D}_{3 / 2}$ level would be $857 \mathrm{~cm}^{-1}$ lower than predicted. In the case of the $152.697 \AA$ line, the energy of the ${ }^{2} \mathrm{D}_{3 / 2}$ level would be instead $136 \mathrm{~cm}^{-1}$ higher than predicted, in closer agreement with the energy of the ${ }^{2} D_{5 / 2}$ level. We note, however, that these levels are strongly mixed as shown in Table 1, so there is some uncertainty in the above arguments.

\subsubsection{Other tentative identifications}

Finally, Table 3 shows that there are several lines that fall around $190 \AA$ and should be observable, although very weak. Several unidentified nickel lines in the EUV were observed by Beiersdorfer et al. (2014), Träbert et al. (2014) with an electron beam ion trap (EBIT), however none of them appear to be due to Ni XII.

Several coronal unidentified lines have also been observed by Del Zanna (2012b) with the Hinode EUV imaging spectrometer (EIS, see Culhane et al. 2007) in an active region. We have assumed the CHIANTI ion fractions and performed an emission measure analysis to estimate the count rates in the Ni XII that are predicted at the Hinode EIS wavelengths for the active region discussed in Del Zanna (2012b). The intensities of the strongest
Ni XII lines are close to the weakest lines observed by EIS, so it is not easy to establish any identifications.

We suggest a few tentative identifications, on the following grounds: 1) the energies are close to those we expect based on the TEC of other levels; 2) the lifetimes of the levels were observed by Trabert et al. (1993) with beam-foil spectroscopy; 3 ) the predicted intensities are close to the observed ones. The decay from level No. $19\left({ }^{2} \mathrm{D}_{5 / 2}\right)$ was observed around $193.3 \AA$ by Trabert et al. (1993). The Hinode EIS spectrum has a weak line at $193.218 \AA$. The decay from level No. $22\left({ }^{2} \mathrm{~F}_{5 / 2}\right)$ was observed around $189.3 \AA$ by Trabert et al. (1993). The Hinode EIS spectrum has a weak line at $189.26 \AA$. The decay from level No. 14 $\left({ }^{4} \mathrm{~F}_{5 / 2}\right)$ was observed around $202 \AA$ by Trabert et al. (1993). The decay from level No. $16\left({ }^{4} \mathrm{P}_{3 / 2}\right)$ was observed around $198.2 \AA$ by Trabert et al. (1993). The Hinode EIS spectrum has a weak line at $198.289 \AA$.

\section{Conclusions}

As in the case of the Cl-like Fe X (Del Zanna et al. 2012), we have found that a large-scale R-matrix calculation for Ni XII produces significant enhancements in the intensities of all the transitions that originate from the lower levels. These results are similar to those we obtained for Fe X (Del Zanna et al. 2012), as briefly outlined in the introduction. They are partly due to resonance enhancement of the collision strengths, and partly due to increased population via cascading from higher levels. We have found similar enhancements for other coronal iron ions.

However, in some cases large differences are not simply due to the resonance enhancement but to the different CI expansion. Overall, the present data provide line intensities that are largely different than previous estimates based on the Matthews et al. (1998) calculations.

For FeX, we were able to use laboratory plates and solar spectra to identify many of the levels of the $3 \mathrm{~s}^{2} 3 \mathrm{p}^{4} 3 \mathrm{~d}$ configuration (Del Zanna et al. 2004). However, for Ni XII this has not been possible. We have suggested several new identifications that have experimental energies close to our estimated ones with the use of the TEC, but they need to be confirmed with more accurate experimental data. Therefore, the adjustments to our calculations via the TEC could be improved in the future if further levels are firmly identified.

We have provided a set of complete atomic data which can be used to aid the identification process using laboratory data. In particular, further high-resolution observations would be useful to clarify the wavelength of the $3 s^{2} 3 p^{5}{ }^{2} \mathrm{P}_{1 / 2}-$ $3 s^{2} 3 p^{4} 3 d^{2} D_{3 / 2}$ transition.

Acknowledgements. The present work was funded by STFC (UK) through the University of Cambridge DAMTP astrophysics grant and the University of Strathclyde UK APAP network grant ST/J000892/1.

\section{References}

Badnell, N. R. 2011, Comp. Phys. Comm., 182, 1528

Badnell, N. R., \& Griffin, D. C. 2001, J. Phys. B Atom. Mol. Phys., 34, 681

Behring, W. E., Cohen, L., \& Feldman, U. 1972, ApJ, 175, 493

Behring, W. E., Cohen, L., Doschek, G. A., \& Feldman, U. 1976, ApJ, 203, 521 Beiersdorfer, P., Träbert, E., Lepson, J. K., Brickhouse, N. S., \& Golub, L. 2014, ApJ, 788, 25

Berrington, K. A., Eissner, W. B., \& Norrington, P. H. 1995, Comput. Phys. Comm., 92, 290

Burgess, A. 1974, J. Phys. B Atom. Mol. Phys., 7, L364

Burgess, A., \& Tully, J. A. 1992, A\&A, 254, 436

Burgess, A., Chidichimo, M. C., \& Tully, J. A. 1997, J. Phys. B Atom. Mol. Phys., 30, 33 
Chidichimo, M. C., Badnell, N. R., \& Tully, J. A. 2003, A\&A, 401, 1177

Culhane, J. L., Harra, L. K., James, A. M., et al. 2007, Sol. Phys., 243, 19

Del Zanna, G. 2012a, A\&A, 546, A97

Del Zanna, G. 2012b, A\&A, 537, A38

Del Zanna, G., \& Badnell, N. R. 2014, A\&A, 570, A56

Del Zanna, G., Berrington, K. A., \& Mason, H. E. 2004, A\&A, 422, 731

Del Zanna, G., Storey, P. J., Badnell, N. R., \& Mason, H. E. 2012, A\&A, 541, A90

Del Zanna, G., Storey, P. J., Badnell, N. R., \& Mason, H. E. 2014, A\&A, 565, A77

Del Zanna, G., Dere, K. P., Young, P. R., Landi, E., \& Mason, H. E. 2015, A\&A, 582, A56

Edlen, B., \& Smitt, R. 1978, Sol. Phys., 57, 329

Fawcett, B. C. 1987, Atom. Data and Nuclear Data Tables, 36, 151

Fawcett, B. C., \& Hatter, A. T. 1980, A\&A, 84, 78

Fawcett, B. C., \& Hayes, R. W. 1972, J. Phys. B Atom. Mol. Phys., 5, 366

Fawcett, B. C., Cowan, R. D., \& Hayes, R. W. 1972, J. Phys. B Atom. Mol. Phys., 5, 2143

Gabriel, A. H., Fawcett, B. C., \& Jordan, C. 1965, Nature, 206, 390

Gabriel, A. H., Fawcett, B. C., \& Jordan, C. 1966, Proc. Phys. Soc., 87, 825

Goldsmith, S., \& Fraenkel, B. S. 1970, ApJ, 161, 317

Griffin, D. C., Badnell, N. R., \& Pindzola, M. S. 1998, J. Phys. B Atom. Mol.

Phys., 31, 3713
Hummer, D. G., Berrington, K. A., Eissner, W., et al. 1993, A\&A, 279, 298 Jefferies, J. T., Orrall, F. Q., \& Zirker, J. B. 1971, Sol. Phys., 16, 103

Keenan, F. P., Botha, G. J. J., Matthews, A., Lawson, K. D., \& Coffey, I. H. 2000, MNRAS, 318,37

Landi, E., Landini, M., Dere, K. P., Young, P. R., \& Mason, H. E. 1999, A\&AS, 135,339

Malinovsky, L., \& Heroux, M. 1973, ApJ, 181, 1009

Matthews, A., Ramsbottom, C. A., Bell, K. L., \& Keenan, F. P. 1998, Atom. Data and Nuclear Data Tables, 70,41

Mattioli, M., Fournier, K. B., Coffey, I., et al. 2004, J. Phys. B Atom. Mol. Phys., 37,13

Nussbaumer, H., \& Storey, P. J. 1978, A\&A, 64, 139

Ryabtsev, A. N. 1979, Sov. Astron., 23, 732

Sandlin, G. D., \& Tousey, R. 1979, ApJ, 227, L107

Sandlin, G. D., Brueckner, G. E., \& Tousey, R. 1977, ApJ, 214, 898

Saraph, H. E. 1978, Comput. Phys. Comm., 15, 247

Sugar, J., Kaufman, V., \& Rowan, W. L. 1992, J. Opt. Soc. Am. B Opt. Phys., 9, 344

Trabert, E., Brandt, M., Doerfert, J., et al. 1993, Phys. Scr, 48, 580

Träbert, E., Beiersdorfer, P., Brickhouse, N. S., \& Golub, L. 2014, ApJS, 215, 6

Wouters, A. W., Schwob, J. L., Suckewer, S., et al. 1988, J. Opt. Soc. Am. B Opt. Phys., 5, 1520

Zeippen, C. J., Seaton, M. J., \& Morton, D. C. 1977, MNRAS, 181, 527 